\title{
Evaluación de un portafolio de títulos de renta variable: modelo para lograr cobertura ante mercados financieros de alta volatilidad
}

\author{
Dr. Nicko Gomero GonZales
}

Docente Asociado de la Facultad de Ciencias Contables

\section{RESUMEN:}

Los mercados bursátiles se muestran inestables, los niveles de riesgo han aumentado considerablemente, algunos especuladores podrían salir ganado en estos escenarios volatilizados, tal como los que hacen operaciones en corto, que son netamente especulativos y que asumen elevados niveles de riesgo, pero la mayoria de inversionistas institucionales salen perjudicados de estos mega problemas financieros, que sin duda van a trascender o impactar en el sector real de la economia. Más claro, los que hagan los especuladores $y$ desestabilizadores de mercados financieros, su efecto transmisión, en este mundo globalizado, se va a sentir en la más lejana economía del mundo. Dada la importancia del mercado bursátil, hay modelos financieros que permiten que los inversionistas orienten adecuadamente sus decisiones de inversión, algunos modelos lo detallaremos y explicaremos en el presente articulo. Además, enfocaremos como objetivo el desarrollo de un modelo estadístico que permita determinar el riesgo y rendimiento de títulos y portafolios de renta variables. Así como explicar un modelo para optimizar decisiones de inversión en el mercado de valores, determinar y explicar los indicadores estadísticos que cuantifiquen el riesgo por inversión en el mercado secundario de valores y determinar la rentabilidad esperada con títulos de renta variable, de distinto nivel de riesgo y con diversos grados de confiabilidad.

Palabras clave: Portafolio, riesgo, diversificación, volatilidad, cobertura, rentabilidad esperada. 


\section{INTRODUCCIÓN}

\section{Marco teórico}

Cuando los mercados financieros se volatilizan es recomendable que los inversionistas tomen posiciones cautelosas, es decir, deben estructurar portafolios que le conlleven a minimizar los riesgos diversificables. Como se conoce, el mercado de valores se mueve más que todo por operaciones especulativas de los agentes financieros institucionales como no institucionales, quienes actúan bajo la línea de obtener elevadas ganancias, «apostando» por la mejor opción de inversión especulativa; más claro, los agentes intervinientes en la Bolsa de Valores desean ganar mucho dinero sólo comprando y vendiendo papeles, los cuales, técnicamente, para fines del presente artículo, los denominaremos documentos de renta variable.

La volatilización de los mercados y su respectivo debilitamiento, como bien se conoce, podría generar serios problemas a la economía real, el efecto transmisión se da por el lado del retorno de las ganancias esperadas, como más que beneficios se transforman en pérdidas, debilitan capitales de muchos negocios e inclusive comprometen fondos que fueron confiados por el público para ser administrados por una institución de inversión.

Este último caso se puede ilustrar de la forma siguiente: si Juan elige una empresa de Fondos de Pensiones para que administre su riqueza que le significará su tranquilidad después de su vida útil en el mercado laboral, esta organización está en la obligación de reportarle ganancias por la inversión de este capital. Si por el contrario, esta institución destina los fondos captados a valores de ele- vada volatilidad, pero a la vez no toma posiciones de cobertura, dada la inestabilidad de los mercados, es casi seguro que Juan al final de una jornada bursátil podría perder su tranquilidad futura y el de su familia. Por decir, si tenía, por ejemplo, S/. 150,000 en la empresa administradora, al final de un día esta riqueza se podría transformar en S/. 135,000 y en una semana de juegos bursátiles podría llegar a convertirse en la mitad de lo que inicialmente tenía. Es decir, Juan perdería la mitad de lo que le iba a representar su pensión de jubilación hasta el final de sus días. ¿Qué falló? Definidamente la empresa administradora no supo estructurar un portafolio que le conllevara a obtener rentabilidades con cobertura para su inversión.

Por otro lado, cabe precisar que la crisis financiera en definitiva implicará a que los inversionistas, más que todo los institucionales, desarrollen nuevos productos financieros. El grado de sofisticación de estos productos estarán dirigidos a buscar rendimientos mayores, pero anteponiendo a ello la mesura y cautela en el proceso de inversión. En definitiva no apostarán en carteras de poca confiabilidad, todo lo contrario, sus decisiones tendrán como soporte, rigurosos estudios fundamentales a fin de que su opción de compra o venta de títulos de renta variable le generen mínimas probabilidades de pérdidas.

Bajo esta línea de trabajo, es altamente probable que se sofistique o en todo caso se perfeccione, por ejemplo, el modelo de Portafolio de Harry Markowitz ${ }^{1}$ o el modelo de Equilibrio de Activos Financieros (CAPM) o en todo caso los analistas buscarán nuevas alternativas al modelo financiero de Sharpe o de Treynor ${ }^{2}$. Es decir, los especuladores actuarán bajo el concepto de mínimos ries-

1 Modelo financiero de optimización de portafolio de valores.

2 Modelos financieros para seleccionar carteras de títulos. 
gos para lograr máximas rentabilidades. Esta manera de proceder en el mercado bursátil quizás vaya contra el principio financiero clásico $^{3}$, situación que significará, como ya se dijo, actitudes menos agresivas de los inversionistas en el mercado bursátil.

Muchos inversionistas institucionales, entre ellos los bancos de inversión, no colocan sus capitales en los mercados especulativos, todo lo contrario, destinan para este fin fondos captados de terceros o agentes superavitarios, ansiosos de lograr rentabilidades superiores a otras alternativas de inversión que podría tener en cartera.

El flujograma para tomar una posición de inversión en valores se presenta en el Gráfico 1. Aquí se puede apreciar claramente cómo los inversionistas deben calificar y clasificar su portafolio, cuya rentabilidad, por cierto, debe superar sus expectativas de ganancias, y si éstas no son las que realmente espera, tendrá que iniciar un proceso de reestructuración, donde va a contrastar dos variables básicas o relevantes: riesgo y rendimiento.

Cabe señalar que las crisis financieras, que algunos analistas califican como coyunturales, han puesto a prueba los modelos de selección de cartera; quizás los modelos matemáticos planteados para este fin sean los correctos, pero sin duda ante una mayor sofistificación de la crisis, amerita que se desarrollen nuevos modelos de selección de portafolios, o en todo caso los tomadores de riesgo deben optimizar el uso de estas herramientas de gestión financiera, de tal forma que, aparte de buscar beneficios, lo cual es natural en todo inversionista, no desequilibren los mercados financieros globales.

A continuación, presentamos un proceso para estructurar portafolios ${ }^{4}$ : $1^{\circ}$ Establecer una política de inversión. Consiste en determinar los objetivos del inversionista y la cantidad de riqueza que está dispuesto a invertir.

$2^{\circ}$ Analizar los valores. Esta fase implica hacer un análisis de valores identificados previamente. Una razón para este análisis es identificar aquellos valores que parezcan estar mal evaluados. Hay varios métodos para el análisis de valores, pero los más importantes y mayormente usados son el análisis técnico y el fundamental.

$3^{\circ}$ Construir la cartera. En esta etapa se construye la cartera, que implica la identificación de acciones específicas en las cuales invertir, así como la determinación de cuánto invertir en cada una, las cuestiones de selectividad y diversificación deben ser tratadas por los inversionistas. La selectividad, también conocida como micropropósito, se refiere al análisis de valores y se enfoca en el pronóstico de los movimientos de precio de valores individuales. El timing, así como el macropropósito, implica el pronóstico de los movimientos de precios de las acciones ordinarias en general respecto de los valores de ingreso fijo, como los bonos corporativos.

$4^{\circ}$ Revisar la cartera. Esta etapa se refiere a la repetición periódica de los tres pasos anteriores. Con el tiempo, el inversionista puede cambiar los objetivos de la inversión, lo que a su vez haría que la cartera actual fuera menos que óptima. El inversionista puede crear una cartera nueva vendiendo ciertos valores y comprando otras.

$5^{\circ}$ Evaluar el desempeño de la cartera. Esta etapa consiste en determinar periódicamente el rendimiento ganado por la cartera y el riesgo que corre el inversionista.

3 Simetría entre el riego y rendimiento, correlación positiva.

4 Tomado de documentos de trabajo de la Maestría de la FCC. Mención Banca y Finanzas - UNMSM. 2008. 
GRÁFICO 1

PROCESO DE ESTRUCTURACIÓN DE UN PORTAFOLIO

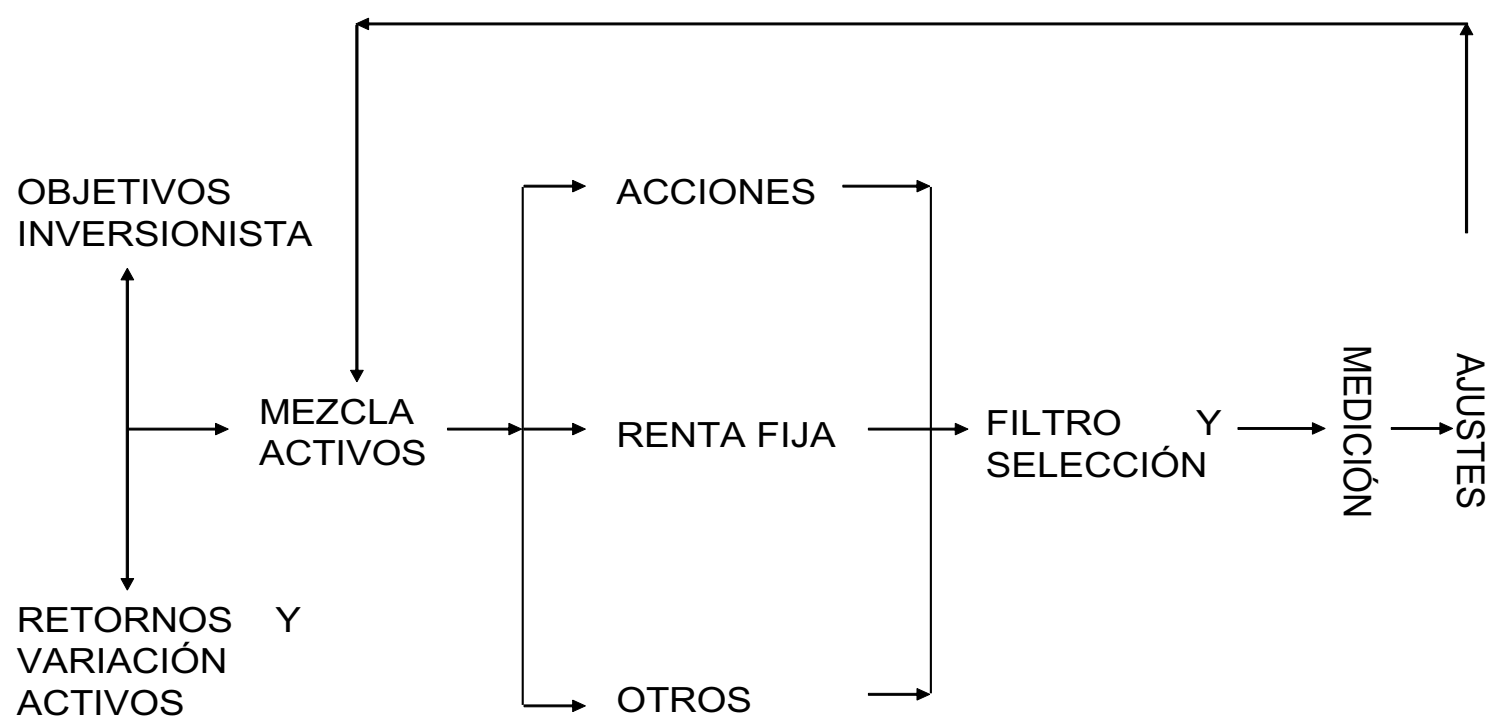

\section{Modelos matemáticos para seleccionar títulos y portafolios de renta variable}

\subsection{Coeficiente de Correlación o de Pearson para optimizar selección de portafolios}

El coeficiente de correlación es un estadígrafo fundamental para tomar decisiones en el mercado bursátil. Como se sabe, este indicador estadístico mide el grado y tipo de relación entre dos variables. El valor del índice de correlación (r) varía en el intervalo $[-1,+1]$ :

- Si $\mathrm{r}=0$, no existe ninguna correlación.

El índice indica, por tanto, una independencia total entre las dos variables, es decir, que la variación de una de ellas no influye en absoluto en el valor que pueda tomar la otra.

- Si $\mathrm{r}=1$, existe una correlación positiva perfecta. El índice indica una dependencia total entre las dos variables denominada relación directa: cuando una de ellas aumenta, la otra también lo hace en idéntica proporción.

- Si $0<\mathrm{r}<1$, existe una correlación positiva.
- Si $\mathrm{r}=-1$, existe una correlación negativa perfecta. El índice indica una dependencia total entre las dos variables llamada relación inversa: cuando una de ellas aumenta, la otra disminuye en idéntica proporción.

- $\mathrm{Si}-1<\mathrm{r}<0$, existe una correlación negativa.

Se dice que un inversionista está perfectamente coberturado cuando mantiene títulos en su cartera inversamente relacionados y cuyo coeficiente de correlación sea igual a -1; por el contrario, si deseara asumir elevados niveles de riesgo demandará títulos con características de simetría perfecta, cuyo coeficiente de correlación debe ser igual a +1 .

Tal como dice la literatura financiera, en el mercado bursátil existen dos tipos de inversionistas, los que asumen elevados niveles de riesgo y los que no son propensos a asumir riesgos elevados para obtener mayores rendimientos. Los primeros mantendrán títulos dentro de su portafolio con un coeficiente de correlación que tienda $a+1$ y el segundo tipo de inversionista, su posición bursátil los lle- 
vará a mantener en cartera activos financieros cuyo coeficiente de correlación tienda a -1 .

Ahora, si el inversionista tiene como propósito diversificar su portafolio a fin de no sacrificar rentabilidades y a la vez no incurrir en riesgos elevados, puede comprar activos directamente e inversamente correlacionados, es decir, la estructura del portafolio estaría conformada por activos con coeficientes de correlación (r) que tiendan a 1 y -1 . En este caso, el presupuesto del inversionista tendrá como destino la compra de estos dos tipos de activos que compensará sus riesgos y a la vez le permitirá obtener rentabilidades que estén por encima de los activos menos riesgosos, sin duda sus beneficios por operaciones especulativas en el mercado de valores le podría generar mejores resultados.

A continuación, en el cuadro 1 , se explica un caso a fin de demostrar la utilidad del coeficiente de correlación en la toma de decisiones bursátiles.

Tal como se puede apreciar en el gráfico, los tres tipos de acciones tienen diferentes grados de volatilidad, por lo que poseen distintos niveles de riesgo. Los más volátiles
CUADRO 1. RENTABILIDAD DE ACCIONES

\begin{tabular}{|l|c|c|c|}
\hline \multirow{2}{*}{ MESES } & \multicolumn{3}{|c|}{ RENTABILIDAD DE LAS ACCIONES } \\
& (Porcentaje) \\
\cline { 2 - 4 } & VOLK & ATK & BNV \\
\hline Enero & 10 & 20 & 19 \\
\hline Febrero & 12 & 15 & 18 \\
\hline Marzo & 15 & -5 & 25 \\
\hline Abril & 10 & 8 & 22 \\
\hline Mayo & 12 & 15 & 30 \\
\hline Junio & 8 & -2 & 15 \\
\hline
\end{tabular}

FUENTE: Elaboración propia

son los que tienen mayor variabilidad, por lo tanto serán más riesgosas, y está representado por el activo ATK

Si no cuenta con un programa estadístico para determinar el valor del coeficiente de correlación puede utilizar la siguiente fórmula:

$$
r=\frac{N \sum X Y-\left(\sum X\right)\left(\sum Y\right)}{\sqrt{N \sum X^{2}-\left(\sum X\right)^{2}} * \sqrt{N \sum Y^{2}-\left(\sum Y\right)^{2}}}
$$

$\mathbf{X}$ como $\mathbf{Y}$ representarían los rendimientos o rentabilidades observados de los títulos a correlacionar.

GRÁFICO 2 COMPORTAMIENTO DE LA RENTABILIDAD DE TRES TIPOS DE ACCIONES: \%

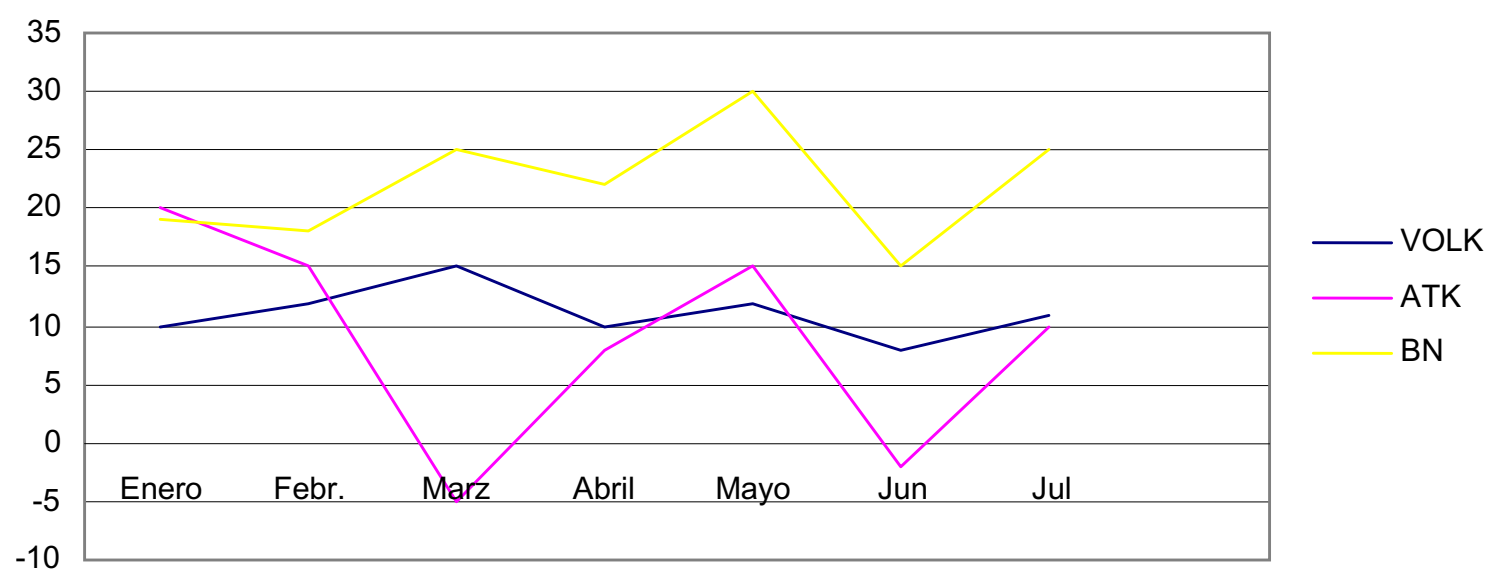


CUADRO 2. COEFICIENTES DE CORRELACIÓN DE TÍTULOS

\begin{tabular}{|l|l|r|r|r|}
\hline \multirow{3}{*}{ VOLK } & Correlación de Pearson & VOLK & ATK & BNV \\
\cline { 2 - 5 } & Sig. (bilateral) & 1 &,- 171 &, 596 \\
\cline { 2 - 5 } & $\mathrm{N}$ & 7 &, 715 &, 158 \\
\hline \multirow{3}{*}{ ATK } & Correlación de Pearson &,- 171 & 7 & 7 \\
\cline { 2 - 5 } & Sig. (bilateral) &, 715 & 1 &, 103 \\
\cline { 2 - 5 } & $\mathrm{N}$ & 7 & &, 827 \\
\hline \multirow{3}{*}{ BNV } & Correlación de Pearson &, 596 &, 103 & 7 \\
\cline { 2 - 5 } & Sig. (bilateral) &, 158 &, 827 & 1 \\
\cline { 2 - 5 } & $\mathrm{N}$ & 7 & 7 & 7 \\
\hline
\end{tabular}

Las cifras se obtuvieron utilizando el programa estadístico SPSS15.

CLASIFICACIÓN DE PORTAFOLIOS

\begin{tabular}{|c|c|c|}
\hline \multirow[b]{2}{*}{$\begin{array}{c}\text { COMBINACIONES } \\
\text { POSIBLES DE ACCIONES }\end{array}$} & \multicolumn{2}{|r|}{ CONDICIÓN DEL PORTAFOLIO } \\
\hline & $\begin{array}{c}\text { COEFICIENTE DE } \\
\text { CORRELACIÓN O } \\
\text { PEARSON }\end{array}$ & \multirow{4}{*}{$\begin{array}{l}\text { De las tres combinaciones posibles de acciones, si el inversionista } \\
\text { adquiere VOL/ATK estará mejor coberturado contra el riesgo, con } \\
\text { relación a las otras dos opciones de inversión. } \\
\text { Ahora, si se tratase de elegir entre la cartera VOLK/BNV y ATK/ } \\
\text { BNV, la primera combinación de acciones será más riesgosa, es } \\
\text { decir, con esta combinación de títulos el inversionista bien podría } \\
\text { ganar más, que adquiriendo ATK/BNV, o en todo caso, también } \\
\text { podría incurrir en mayores pérdidas. }\end{array}$} \\
\hline VOLK/ATK & $-0,171$ & \\
\hline VOLK/BNV & 0,596 & \\
\hline ATK/BNV & 0,103 & \\
\hline
\end{tabular}

Como se puede apreciar en el Cuadro 2, el coeficiente de correlación puede conducir al inversionista a tomar una decisión acertada en cuanto al riesgo y rendimiento de sus activos, es decir, utilizando este estadígrafo puede ejecutar una buena selección de portafolios.

\subsection{Selección de títulos utilizando la varianza o la desviación típica}

Así como el coeficiente de correlación es una buena herramienta para tomar decisiones en el mercado bursátil, la desviación típica es otra alternativa para dicho propósito. En este caso, este estadígrafo medirá el grado de volatilidad de un título, es decir, cuantificará su nivel de riesgo, por lo que el inversionista podrá elegir títulos calificándolos según su grado de sensibilidad, orientando su posición hacia aquellas que le den más rentabilidad a costa de incurrir en mayores riesgos o, en todo caso, tendrá una actitud mucho más mesurada en cuanto a la acción elegida, o sea, su propensión será de tomar títulos con bajas desviaciones típicas o varianzas.

La desviación típica no sólo es aplicable para títulos en forma excluyente, sino que, además, se puede aplicar para una cartera, por lo que al determinar su nivel de volatilidad en términos cuantitativos, justamente a través de la desviación típica, se habrá determinado el riesgo del portafolio o de la cartera de títulos.

La regla es la siguiente: cuanto más volátil es un título, mayor será su desviación típica, por lo que el inversionista al adquirir activos con estas características exigirá una mayor rentabilidad a su inversión realizada. La misma regla se aplica a portafolios de activos financieros.

Cabe precisar que el inversionista necesita estimar los rendimientos esperados y las

\section{QVIPVIRAMAYOC}


varianzas de todos los valores contemplados. Además, necesita estimar todas las covarianzas entre estos valores. Una vez cuantificados estos estadígrafos, estará en capacidad de identificar la composición de la cartera así como su rendimiento esperado

Los inversionistas evalúan las carteras juzgando los rendimientos esperados y desviaciones estándares de las carteras durante un horizonte de tiempo, en este sentido, nunca se sacian, de modo que cuando se da una opción entre dos carteras con desviaciones estándar idénticas, elegirán la que tenga el rendimiento esperado más alto (criterio de elección de la media varianza: máximo rendimiento).
Así como los inversionistas desean obtener máximas ganancias, también presentan actitudes de no querer asumir mayores riesgos, por ello, cuando se da una opción de elegir entre dos carteras con rendimientos esperados idénticos, elegirán las que tengan la desviación estándar más baja (criterio de elección de la media varianza: mínimo riesgo).

En sí, la desviación típica es una herramienta útil para priorizar o seleccionar títulos o en todo caso elegir portafolios en función del riesgo o volatilidad que proyecta.

A fin de validar el uso de la desviación típica, se obtendrá los resultados para este indicador de la rentabilidad de los títulos expuestos en el Cuadro 2.

Cuadro 3. DESVIACIÓN TíPICA DE LOS TÍTULOS

\begin{tabular}{|l|r|r|r|r|r|}
\hline & N & \multicolumn{1}{|c|}{ Mínimo } & \multicolumn{1}{c|}{ Máximo } & \multicolumn{1}{c|}{ Media } & \multicolumn{1}{c|}{ Desv. típ. } \\
\hline VOLK & 7 & 8,00 & 15,00 & 11,1429 & 2,19306 \\
\hline ATK & 7 & $-5,00$ & 20,00 & 8,7143 & 9,23245 \\
\hline BNV & 7 & 15,00 & 30,00 & 22,0000 & 5,09902 \\
\hline N válido (según lista) & 7 & & & & \\
\hline
\end{tabular}

Media: rentabilidad promedio esperada por cada tipo de título.

Desviación típica: indicador de riesgo o volatilidad para cada tipo de título.

Por la información observada se puede afirmar que la acción más riesgosa es ATK, ya que su coeficiente de volatilidad llega al $9,23 \%$. Por el contrario, VOLK es el título menos riesgoso, por ello la ganancia del inversionista será moderada. En este caso se está suponiendo que la demanda de títulos es en forma excluyente, es decir, el inversionista bien podría comprar acciones riesgosas o en su defecto adquirir acciones de riesgos moderados, pues no estructura un portafolio.

La desviación típica también se podría determinar utilizando la siguiente fórmula:

$$
: \sum \frac{(X i-X)^{2}}{\mathrm{n}-1}
$$

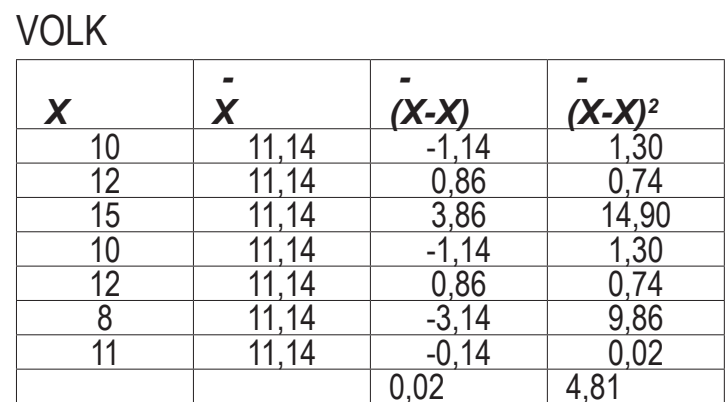

Varianza: 4,81\% / Desviación típica: 2,19\%

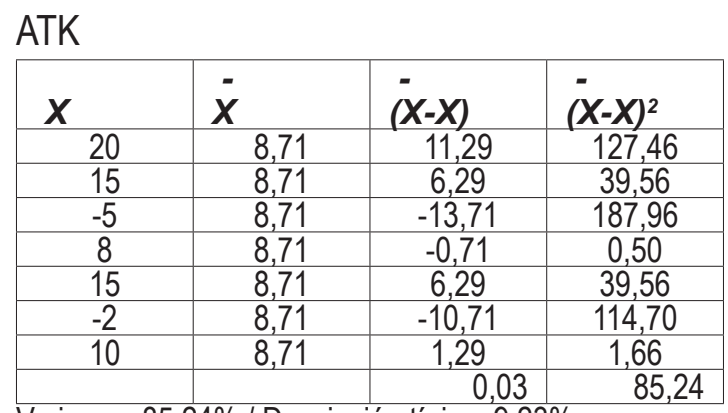

Varianza: 85,24\% / Desviación típica: 9,23\% 
BNV

\begin{tabular}{|c|c|c|c|}
\hline$x$ & $\bar{x}$ & $\overline{(X-X)}$ & $\overline{(X-X)^{2}}$ \\
\hline 19 & 22 & -3 & 9,00 \\
\hline 18 & 22 & -4 & 16,00 \\
\hline 25 & 22 & 3 & 9,00 \\
\hline 22 & 22 & 0 & 0,00 \\
\hline 30 & 22 & 8 & 64,00 \\
\hline 15 & 22 & -7 & 49,00 \\
\hline 25 & 22 & 3 & 9,00 \\
\hline & & 0.0 & 26.0 \\
\hline
\end{tabular}

Los indicadores de riesgo, mediante esta metodología, coinciden exactamente, tal como se obtuvo utilizando el programa estadístico SPSS15. Al margen de la coincidencia, la acción más volátil es ATK, situación que se refleja en los indicadores estadísticos (varianza y desviación típica para este tipo de documento).

Ahora, si el inversionista deseara saber la rentabilidad que obtendría al invertir en estos tipos de acciones, podría emplear la siguiente fórmula:

\section{$R e=$ rentabilidad media $+/-$ desviación típica de la acción}

\section{Rentabilidad esperada de VOLK}

Re: $11,14+2,19=13,33 \%$

Re: $11.14-2,19=6,76 \%$

Rentabilidad esperada de ATK

Re: $8,71+9,23=17,94 \%$

Re: $8,71-9,23=-0,52 \%$

Si el inversionista compra la acción ATK, bien podría ganar hasta $17,94 \%$ o, en todo caso, perder $-0,54 \%$. Como VOLK es menos riesgosa, la ganancia sería más moderada. La rentabilidad obtenida es para un $68 \%$ de nivel de confianza.

CLASIFICACIÓN DE TÍTULOS SEGÚN EL NIVEL DE RIESGO

\begin{tabular}{|l|c|c|c|l|}
\hline TítULOS & $\begin{array}{c}\text { ReNTABILIDAD MEDIA } \\
\%\end{array}$ & $\begin{array}{c}\text { VARIANZA } \\
\%\end{array}$ & $\begin{array}{c}\text { DeSVIACIÓN TÍPICA } \\
\%\end{array}$ & \multicolumn{1}{c|}{ CALIFICACIÓN } \\
\hline ATK & 8,71 & 85,24 & 9,23 & Riesgosa \\
\hline BNV & 22,0 & 26,0 & 5,09 & Riesgo moderado \\
\hline VOLK & 11,14 & 4,81 & 2,19 & Bajo riesgo \\
\hline
\end{tabular}

\section{CONCLUSIONES}

Los mercados financieros altamente volatilizados exigen que los inversionistas utilicen mecanismos de cobertura para proteger sus inversiones en el mercado de valores.

Existen modelos financieros bajo el soporte del instrumental estadístico, que señalan la ruta crítica para que el inversionista elija la mejor opción de inversión en los mercados especulativos.

El coeficiente de correlación es un instrumental estadístico que permite al inversionista elegir portafolios sin riesgo o con él, así como priorizar sus decisiones en el mercado bursátil. Para darle mayor consistencia a este resultado se puede utilizar la covarianza, herramienta estadística que señala el grado de correlación entre dos variables analizadas.

Asimismo, la desviación típica es un indicador de volatilidad, por lo que las acciones de mayor grado de riesgo presentarán cifras elevadas. Para determinar la rentabilidad esperada, la desviación típica es considerada como una prima por riesgo, por lo que las expectativas de ganancias de un inversionista serán mayores cuanto más grande sea este indicador de volatilidad. 


\section{REFERENCIAS}

Bodie-Merton. Finanzas. 1a. ed., México, Prentice-Hall, 2003.

Brealey, Richard y Stewart Myers. Principios de finanzas corporativas, 3 a. ed., Caracas, McGraw-Hill, 1992.

Brigham, Eugene y Joel Hosuton. Fundamentos de administración financiera. 10ma. ed., España, Edit. Thomson, 2006.

Scott Besley, Eugene F. Brigham. Fundamentos de administración financiera 12a ed., McGraw-Hill, 2005.

Ross, Stephen; Westerfield, Randolph W. y Jeffrey Jaffe. Finanzas corporativas. 7a. ed., España, McGraw-Hill, 2005. 